\title{
La Reichsbank, 1876-1920. Une analyse institutionnelle et cliométrique
}

Olivier Darne et Claude Diebolt

\section{(2) OpenEdition}

\section{Journals}

Édition électronique

URL : http://journals.openedition.org/ress/233

DOI : $10.4000 /$ ress.233

ISSN : 1663-4446

Éditeur

Librairie Droz

Édition imprimée

Date de publication : 1 juillet 2007

Pagination : 203-212

ISBN : 978-2-600-01155-6

ISSN : 0048-8046

Référence électronique

Olivier Darne et Claude Diebolt, « La Reichsbank, 1876-1920. Une analyse institutionnelle et cliométrique », Revue européenne des sciences sociales [En ligne], XLV-137 | 2007, mis en ligne le 01 juillet 2010, consulté le 30 avril 2019. URL : http://journals.openedition.org/ress/233 ; DOI : 10.4000/ ress.233 
Olivier DARNÉ et Claude DIEBOLT

\section{LA REICHSBANK, 1876-1920 Une analyse institutionnelle et cliométrique}

\section{INTRODUCTION}

En 1870, la condition de mise en place du système monétaire* reflète la division politique de l'Allemagne. Sept systèmes, fondés sur le Standard Argent, sont en vigueur en même temps, trente deux banques et vingt deux Etats mettent un très grand nombre de billets en circulation. Cette confusion monétaire résulte du développement économique des années 1840 et 1850, ainsi que de la création des banques d'émission (Zettelbanken). En fait, sur les vingt deux Etats allemands, seule la Prusse a un système monétaire bien organisé et régulé par la Banque de Prusse (Preussische Notenbank, qui trouve son origine dans la Königliche Giround Lehnbank).

La première tentative en direction d'un meilleur système monétaire est prise en mars 1870. Une loi est votée, impliquant que le privilège d'émission de billets peut uniquement être obtenu avec l'autorisation fédérale. Cette loi a été élaborée comme une mesure provisoire jusqu'à ce qu'une solution permanente puisse être trouvée et être acceptée par toutes les parties concernées. Pour ce faire, Bismarck suggère, en 1872, une loi qui transforme la Banque de Prusse en Banque centrale de l'Allemagne. Suite à l'opposition farouche du Ministre des Finances prussien, la proposition est retirée avant même d'avoir été présentée au Reichstag. Deux ans plus tard, une nouvelle loi est présentée. Désormais, le projet de création d'une Banque centrale devient une visée acceptable.

Le 22 septembre 1875, l'unification monétaire de l'Allemagne est décrétée. A partir du $1^{\text {er }}$ janvier 1876, le système monétaire entre en vigueur sur l'ensemble du territoire ${ }^{1}$. La réforme bancaire érige la banque de Prusse en banque centrale de l'Empire, avec la nouvelle appellation de Reichsbank, chargée de fournir des crédits au gouvernement impérial et de mener une politique active du taux de l'escompte.

Pour des raisons liées à la conjoncture économique, la Reichsbank doit, avant la Première Guerre mondiale, plusieurs fois se départir de sa réserve statutaire. La période de 1876 à 1894, par exemple, débute par une très longue récession (de

* Cet article s'intègre dans un projet de recherche de l'Association Française de Cliométrie (AFC), intitulé « Monnaie et croissance en Allemagne aux XIX ${ }^{\mathrm{e}}$ et $\mathrm{XX}^{\mathrm{e}}$ siècles ».

1 Pour le lecteur intéressé par une analyse institutionnelle approfondie, voir Darné et Diebolt (2001). 
6 ans environ), suivie d'une faible reprise, insuffisante pour surmonter une nouvelle dépression, de 1883 à 1887, marquée par un chômage important et une baisse du revenu de larges couches de la population. Pendant ces années, les banques privées ont tenté de prendre une position prépondérante sur le marché monétaire. La Reichsbank est alors contrainte de pratiquer l'escompte au-dessous de son propre taux afin de conserver le contrôle du marché en période d'excessive liquidité. Il en est ainsi jusqu'en 1896. Les cinq dernières années du siècles sont, en revanche, une période d'extraordinaire expansion pour l'Allemagne. Malgré les courtes dépressions de 1901 et de 1908, il en est de même jusqu'en 1913. En fait, l'économie allemande devient de plus en plus dépendante de l'extérieur. Sa balance des paiements courants demeure pourtant positive grâce aux excédents de la balance des services notamment. A cette époque, la Reichsbank ne craint donc aucune tension sur le Mark provoquée par les sorties d'or. La conjoncture générale est favorable, les bénéfices des entreprises augmentent très rapidement et ceci malgré le renchérissement des matières premières et des produits alimentaires. Cela provient, pour l'essentiel, d'un progrès technique intense et d'une organisation économique et commerciale très dynamique, fondée sur une forte concentration verticale et horizontale, ainsi qu'une étroite liaison entre les secteurs industriels et bancaires. Progressivement, le Mark devient une des monnaies-or les plus solides du monde ! Mais, il faut aussi préparer la guerre d'un point de vue financier. Ainsi, dès 1911 , on perçoit une sorte de convergence des réformes économiques et financières vers un but politique déterminé. A cette époque, la préparation monétaire à la guerre consiste à discipliner les banques pour qu'elles constituent des encaisses plus élevées, à persuader le public d'utiliser le chèque plutôt que la monnaie métallique (pour ne pas mettre les réserves d'or à contribution) et, pour la Reichsbank, à accroître ses avoirs en or, à accaparer tout l'or à la limite pour ne laisser circuler que du papier. Telle est, en tout cas, la préoccupation de la loi qui autorise l'émission de billets de 20 et de 50 marks, et celle des industriels patriotes qui se mettent à payer systématiquement leurs ouvriers en petits billets. Notables et commerçants de Berlin vont suivre l'exemple, les administrations également, avec comme ambition, croyait-on, de contribuer à l'amélioration de l'armement de guerre. En somme, une sorte de trésor de guerre, dont Hume parlait déjà ironiquement au XVIII ${ }^{\mathrm{e}}$ siècle, en référence à l'argent accumulé par le roi-sergent, Frédéric-Guillaume Ier. Pourtant, le résultat de cet ensemble de mesures a été assez extraordinaire. Le dernier bilan de paix de la Reichsbank, celui du 23 juillet 1914, dépasse tout ce que l'on peut espérer, puisque pour la première fois la monnaie en circulation est couverte à $90 \%$ par l'encaisse, sans compter l'appoint du trésor de guerre. La marge d'émission non imposable dépasse alors les 3 milliards de francs. La mobilisation financière s'opère donc aussi aisément que la mobilisation militaire (dès l'ultimatum de l'Autriche-Hongrie à la Serbie). La Reichsbank est prête à jouer son rôle de «banque de guerre » et ceci jusqu'au moment où l'or russe est exigé à Brest-Litovsk. Suit la dépréciation accélérée, la faillite totale du Mark (après le premier conflit mondial) ; un événement de caractère unique dans l'histoire monétaire. Elle correspond à une période d'opposition politique violente, de grande misère, mais aussi d'enrichissement pour les investisseurs avisés.

Partant de là, nous proposons, en prolongement d'un travail précédent (Darné et Diebolt, 2000), une analyse cliométrique de la série mensuelle de la réserve 
monétaire de la Reichsbank, couvrant la période allant de janvier 1876 à décembre 1920. Dans un premier temps, nous appliquons différents tests de non stationnarité afin de déterminer la nature déterministe ou stochastique de la série. Dans un second temps, nous réalisons l'analyse de la tendance (tendance et cycle) à partir de l'approche des modèles de séries temporelles structurels, ceci afin d'identifier les points de retournement de notre série statistique.

Figure 1: Série mensuelle de la réserve monéaire de la Reichsbank

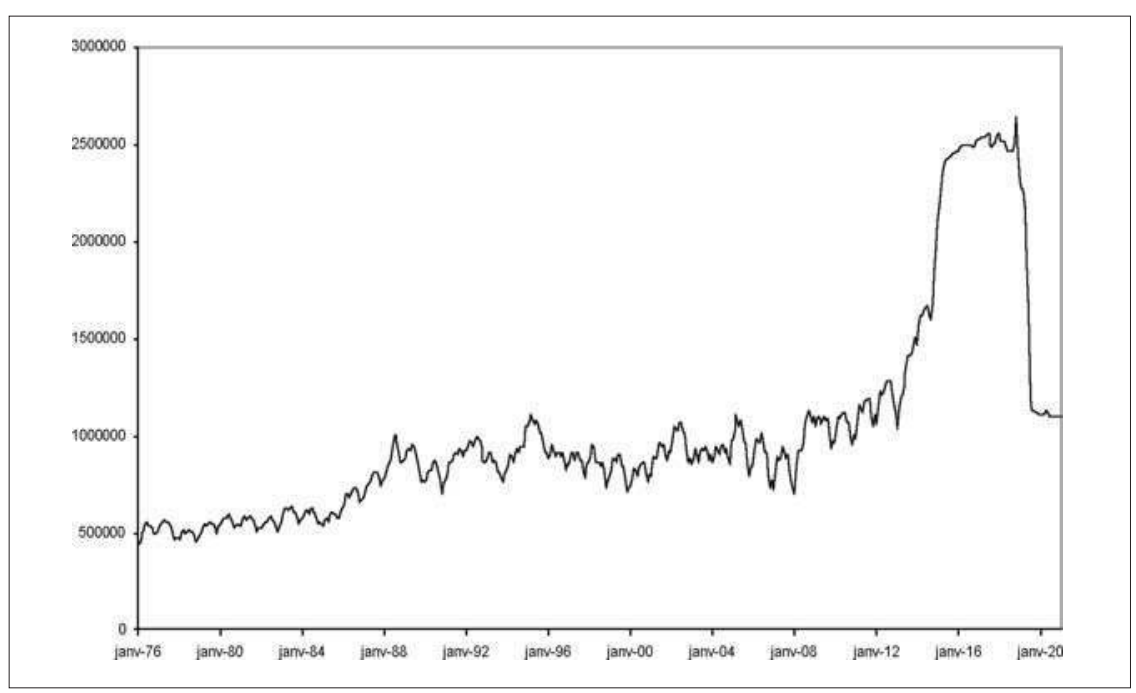

\section{LES TESTS DE NON STATIONNARITÉ}

Nous utilisons divers tests de non stationnarité sur la série de la réserve monétaire de la Reichsbank afin de savoir si la non stationnarité de la composante de tendance est de nature déterministe ou stochastique. Nous appliquons les tests de racine unitaire efficients de Elliott et alii (1996) et de Ng et Perron (2001), ainsi que le test de racine unitaire avec rupture structurelle de Zivot et Andrews (1992) puisque la série affiche clairement une rupture avant la Première Guerre mondiale. Ces tests vont nous permettre de savoir si la série possède une racine unité, c'est-à-dire si notre chronique suit un processus stochastique non-stationnaire. Nous les combinons avec le test de stationnarité de Kwiatkowski et alii (1992) afin de confirmer les résultats des tests précédents et ainsi obtenir des résultats plus robustes.

\subsection{Le test de ERS (1996)}

Elliott, Rothenberg et Stock (1996) [ERS] ont développé un test de point optimal de racine unitaire qui repose sur une quasi-différenciation de la série $X_{t}$. Cette approche permet d'améliorer la puissance du test par rapport aux tests standards de Dickey et Fuller $(1979,1981)$. 
On définit :

$$
\begin{aligned}
& Y_{t}=X_{t}-\alpha X_{t-1} \\
& y_{t}=z_{t}-\alpha z_{t-1}
\end{aligned}
$$

où $z_{t}$ vaut 1 dans le cas sans dérive, $(1, t)$ dans le cas avec dérive, et $a=1-(c / T)$ avec $c$ égale à 7 si la série ne semble pas contenir de tendance (dérive) et à 13.5 si elle semble en contenir une. On régresse $Y_{t}$ sur $y_{t}$ par les Moindres Carrés Généralisés (MCG), et on calcule la variable :

$$
Z_{t}=X_{t}-\psi^{\prime} z_{t}
$$

où $y$ est l'estimateur des MCG de la régression précédente. On effectue ensuite la régression suivante :

$$
\Delta Z_{t}=\beta_{0} Z_{t-1}+\sum_{j=1}^{k} \beta_{j} \Delta Z_{t-j}+\varepsilon_{t}
$$

Les statistiques de test proposées ${ }^{2}, E R S_{r}$ (sans dérive) et $E R S_{t}$ (avec dérive), sont les $t$-statistiques de Student pour l'hypothèse nulle $\beta_{0}=0$. La règle de décision est la suivante : si $E R S>E R S_{\text {tab }}$, alors $H_{0}$ est acceptée et la série est non stationnaire, sinon elle est rejetée.

\subsection{Le test de Ng et Perron (2001)}

Perron et $\mathrm{Ng}$ (1996) ont proposé une version modifiée des tests de Phillips et Perron (1988) qui sont plus robustes aux distorsions de taille lorsque les résidus ont une corrélation sérielle négative. Ces tests, $M Z_{r}$ et $M Z_{t}$, sont nommés les $M$ tests et sont définis par:

$$
\begin{gathered}
M Z_{\rho}=Z_{\rho}+(T / 2)(\hat{\phi}-1)^{2} \\
M Z_{\rho}=\left(X_{T}^{2}-T s_{a r}^{2}\right)\left(2 T^{-1} \sum_{t=1}^{T} X_{t}^{2}\right)^{-1} \\
M Z_{\tau}=Z_{\tau}+(1 / 2)(\hat{\phi}-1)^{2}\left(\sum X_{t-1}^{2} / s_{a r}^{2}\right)^{1 / 2} \\
M Z_{\tau}=\left(X_{T}^{2}-T s_{a r}^{2}\right)\left(2 T^{-1} \sum_{t=1}^{T} X_{t}^{2}\right)^{-1}\left(T^{-2} \sum X_{t-1}^{2} / s_{a r}^{2}\right)^{1 / 2}
\end{gathered}
$$

A l'instar de Elliott et alii (1996) qui montrent que les données detrended par les MCG donnent des gains de puissance pour le test de Dickey-Fuller sur le test de Dickey-Fuller standard, Ng et Perron (2001) proposent de considérer les $M$ -

2 Ces statistiques de test sont aussi nommées statistiques MCG demeaned et detrended de Dickey-Fuller (MCG-DF). 
tests sous cette approche. Ces $M^{M C G}$-tests, notés $M Z_{r}^{M C G}$ et $M Z_{t}^{M C G}$, confirment leur puissance par rapport aux $M$-tests.

\subsection{Le test de Zivot et Andrews (1992)}

Zivot et Andrews (1992) ont développé un test de racine unitaire avec une rupture structurelle introduite de manière « endogène », c'est-à-dire que le point de changement (inconnu) est estimé plutôt que fixé.

Ils considèrent l'hypothèse nulle de racine unitaire sans rupture structurelle exogène, et l'hypothèse alternative d'un processus stationnaire en tendance avec un changement dans la tendance à un moment inconnu du temps $T_{B}\left(1<T_{B}<T\right)$.

Zivot et Andrews (1992) régressent l'équation de régression suivante :

$$
X_{t}=\mu+\delta D U_{t}(\lambda)+\beta t+\alpha X_{t-1}+\sum_{j=1}^{k} c_{j} \Delta X_{t-j}+\varepsilon_{t}
$$

où $D U_{t}(l)=1$ si $t>T \lambda, 0$ sinon, et $\lambda=T_{B} / T$ est la localisation du point de rupture.

Puisque la rupture structurelle est endogène, on utilise la statistique de DickeyFuller minimum pour tester la présence d'une racine unitaire, et on rejette l'hypothèse nulle d'une racine unitaire si :

$$
\inf _{\lambda} t_{\alpha}(\lambda)<\kappa_{\text {inf }, \alpha}
$$

où $k_{i n f, \alpha}$ représente la valeur critique de inf $t_{\alpha}(l)$.

\subsection{Le test de KPSS (1992)}

Kwiatkowski et alii (1992) [KPSS] ont proposé un test de stationnarité qui prend en considération l'existence éventuelle d'autocorrélations des résidus d'une série temporelle $X_{t}$.

Cette méthode teste l'hypothèse nulle de stationnarité en niveau (test- $\mu$ ) ou autour d'une tendance (test- $t$ ) contre l'hypothèse alternative d'une racine unitaire.

On régresse $X_{t}$ sur une constante (test- $\mu$ ) ou sur une constante et une tendance (test- $t$ ), et on détermine le résidu estimé, $\hat{e}_{t}$ :

$$
X_{t}=\alpha+\beta t+e_{t}
$$

La statistique de test est :

$$
\operatorname{KPSS}_{\tau / \mu}=\frac{1}{s_{w a}^{2}} \frac{\sum_{t=1}^{T} \hat{S}_{t}^{2}}{T^{2}}
$$

où $\hat{S}_{t}=\sum_{t i=1}^{t} \hat{e}_{t}(t=1, \ldots, T)$ est la somme partielle des résidus, et est l'estimation ue ra valialıce de long terme de $\hat{e}_{t}$.

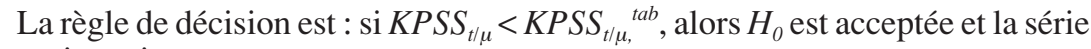
est stationnaire. 
Les résultats des tests de racine unitaire efficients ainsi que ceux avec rupture structurelle montrent que la série de la réserve monétaire de la Reichsbank possède une racine unitaire. Ce constat est confirmé par le test de KPSS qui rejette l'hypothèse nulle que la série soit stationnaire (en niveau ou en tendance), et par le test de Dickey-Pantula qui détecte une seule racine unitaire. Par conséquent, la composante de tendance de la série suit un processus non stationnaire stochastique, c'est-à-dire une marche aléatoire. Du point de vue des implications économiques, ce résultat signifie que les chocs aléatoires, c'est-à-dire des chocs technologiques, qui ont affectés l'économie ont eu un effet permanent sur la série, à savoir qu'elle n'a pas retrouvé sa trajectoire d'origine.

\section{TENDANCE, CYCLE ET POINTS DE RETOURNEMENT}

L'analyse de la tendance est réalisée à partir de l'approche des modèles de séries temporelles structurels (STS), ou de composantes inobservables (voir Harvey, 1989 et 2000).

Un modèle STS est fondé sur la formulation directe de modèles pour chaque composante dans la décomposition de la série d'origine $X_{t}$ de la manière suivante:

$$
X_{t}=T_{t}+S_{t}+C_{t}+I_{t}
$$

où $T_{t}, S_{t}, C_{t}$ et $I_{t}$ sont respectivement les composantes de tendance, saisonnière, cyclique et irrégulière. La composante irrégulière est généralement supposée être un bruit blanc Gaussien de moyenne zéro et d'écart-type $\beta_{\varepsilon}^{2}$, noté $\varepsilon_{\mathrm{r}} \sim \mathrm{N}\left(0, \beta_{\varepsilon}^{2}\right.$.

La tendance est généralement modélisée comme une marche aléatoire avec dérive, où la dérive est fixée. Nous retenons cette modélisation au vu des résultats antérieurs. Formellement, nous avons :

$$
T_{t}=T_{t=1}+\beta_{t}+\eta_{t}
$$

où $\eta_{t} \sim N\left(0, \sigma_{\eta}^{2}\right)$ est un bruit blanc Gaussien, et et sont non corrélés.

La composante cyclique est de type stochastique. Elle est modélisée de la manière suivante :

$$
\left[\begin{array}{c}
C_{t} \\
C_{t}^{*}
\end{array}\right]=\rho\left[\begin{array}{cc}
\cos \lambda_{c} & \sin \lambda_{c} \\
-\sin \lambda_{c} & \cos \lambda_{c}
\end{array}\right]\left[\begin{array}{l}
C_{t-1} \\
C_{t-1}^{*}
\end{array}\right]+\left[\begin{array}{l}
\kappa_{c, t} \\
\kappa_{c, t}^{*}
\end{array}\right]
$$

où $t=1, \ldots, T, \rho$ est un facteur d'amortissement $(0<\rho<1), \lambda_{c}$ est la fréquence $\left(0<\lambda_{c}<\pi\right)$; et $\kappa_{c, t}^{*}$ sont deux bruits blancs indépendants entre eux, de moyenne zéro et de variance commune $\sigma_{k}^{2}$. La période du cycle est égale à $2 \pi / \lambda_{c}$. Il s'agit de la durée théorique, c'est-à-dire le temps nécessaire pour qu'une oscillation cyclique complète ait lieu à la suite d'une impulsion initiale. 
Puisque nous avons montré la présence de fluctuations saisonnières déterministes dans la série de la réserve monétaire de la Reichsbank (voir Darné et Diebolt, 2000) la composante saisonnière est modélisée sous forme de variables dichotomiques :

$$
S_{t}=-\sum_{j=1}^{s-1} S_{t-j}+\omega_{t} \quad \text { avec } \omega_{t} \sim N\left(0, \sigma_{\omega}^{2}\right)
$$

L'estimation des différentes composantes est obtenue en utilisant le filtre de Kalman. Les calculs sont réalisés en utilisant le logiciel STAMP (Structural Time Series Analyser, Modeller and Predictor) développé par Koopman et alii (1995). Nous obtenons une périodicité du cycle de 15.2, soit 1.27 années.

Nous déterminons ensuite les points de retournement. Pour ce faire, nous considérons qu'un point de retournement survient en un point $t$ dans le temps lorsqu'une des observations d'une série $y_{t}$ a une valeur plus élevée (ou plus faible) que les $k$ observations précédentes et les $m$ observations subséquentes (ou égales à celles-ci), c'est-à-dire :

passage à une phase de récession : $y_{t-k} \leq \ldots \leq y_{t-1}>y_{t} \geq y_{t+1} \geq \ldots \geq y_{t+m}$ passage à une phase d'expansion : $y_{t-k} \geq \ldots \geq y_{t-1}<y_{t} \leq y_{t+1} \leq \ldots \leq y_{t+m}$

A ce jour, il n'existe pas de consensus concernant les valeurs de $k$ et $m$ afin de définir un point de retournement dans le cadre de données mensuelles de la tendance. A l'instar de Dagum (1996), nous considérons néanmoins qu'un point de retournement se produit quand $k=3$ et $m=1$.

Les résultats sont présentés dans le tableau 2. Ils montrent, à l'image de la figure 3, que le mouvement général de la réserve monétaire de la Reichsbank a

Figure 2: Tendance de la réserve monétaire de la Reichsbank

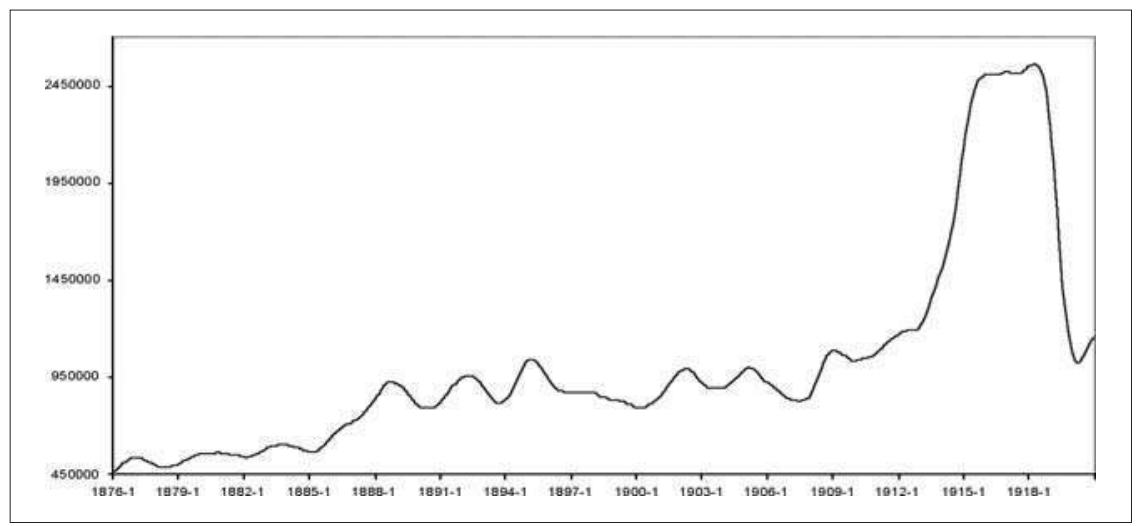

3 Voir également Zellner, Hong et Min (1991), LeSage (1991) et Pfeffermann et Bleuer (1992). 
Figure 3: Cycle de la réserve monétaire de la Reichsbank

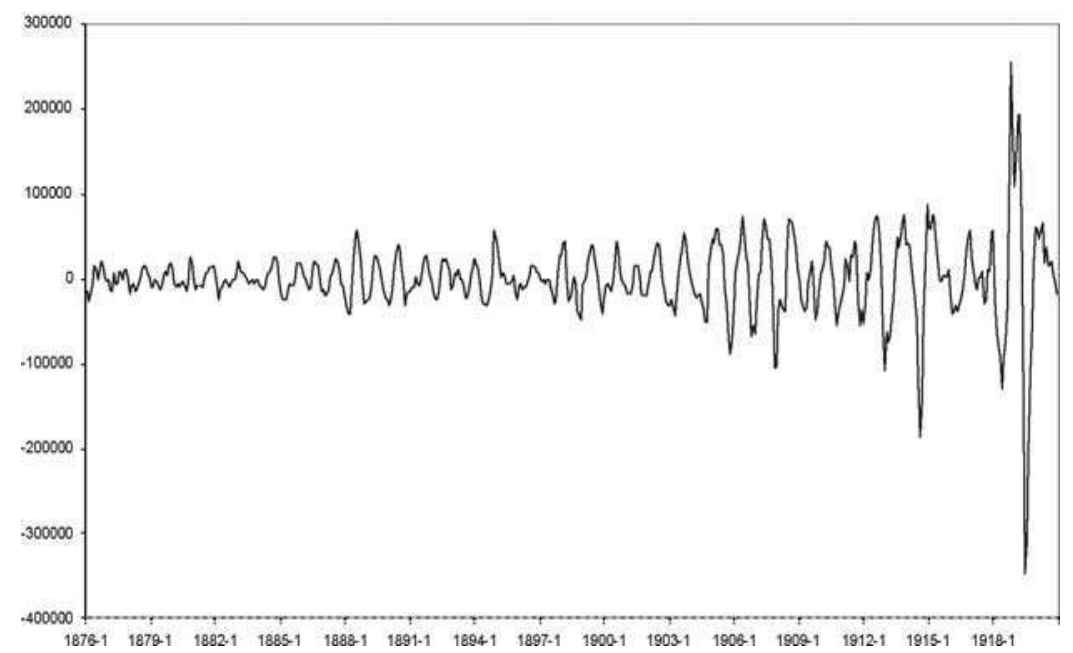

pris une allure rythmique, en suivant une cadence nettement marquée. Les phases de prospérité et de dépression alternent avec une relative régularité. Le mouvement est périodique, non rigoureusement cyclique, à amplitude croissante, augmentant au fur et à mesure que la tendance se prolonge. Dans le cadre de notre fenêtre statistique, nous constatons que, dix fois, la réserve monétaire de la Reichsbank a été violemment perturbée. Les crises se sont produites aux dates suivantes : 1877, 1880, 1883, 1888, 1892, 1895, 1902, 1905, 1909 et 1918. La liste de ces années critiques suscite l'idée que la crise était générale ou généralisée dans l'espace. Par ailleurs, l'intervalle entre deux crises consécutives n'a varié qu'entre des limites assez rapprochées, comme le montrent nos calculs cliométriques.

\section{CONCLUSION}

En guise de conclusion, nous dirons que l'analyse institutionnelle et cliométrique de la réserve monétaire de la Reichsbank conduit à définir des équilibres non plus stationnaires, mais temporellement emboîtés, faisant apparaître des cycles ou des bifurcations, c'est-à-dire des mouvements alternatifs caractérisés à la fois par leur régularité et leur amplitude. Le cycle est un phénomène d'onde, sans nécessairement suivre la régularité d'une courbe sinusoïdale. Par ailleurs, le cycle ne peut pas être défini comme un pur phénomène de répétition. Il ne doit pas non plus être perçu comme un phénomène unique car, dans ce cas, le problème de la cyclicité disparaîtrait en tant que tel. Ce ne sont donc pas les situations stables et les permanences qui nous intéressent, mais les évolutions, les crises et les instabilités.

L'histoire économique nous montre le relativisme de ces mouvements, qui ne se trouvent pas nécessairement dans tous les systèmes économiques, ni dans tous 
les pays. Certains sont caractéristiques d'une époque, d'autres d'une économie. En fait, chaque mouvement tire une partie de sa forme et de ses particularités du mouvement plus fondamental qui le sous-tend. Ainsi, la nature des fluctuations dépend du système socio-économique qui les engendre, tout en sachant que leurs causes peuvent varier au cours de l'histoire, en fonction de la structure économique du pays. Les tendances séculaires synthétisent l'évolution fondamentale des structures socio-économiques sur lesquelles se superposent toutes les autres fluctuations.

Tableau 1 : Tests de non stationnarité

\begin{tabular}{|c|c|c|}
\hline Tests & Estimation & Valeur critique \\
\hline$E R S_{\rho}$ & -0.42 & -1.98 \\
$M Z_{r}^{M C G}$ & -0.71 & -8.10 \\
$M Z_{\tau}^{M C G}$ & -0.45 & -1.98 \\
$Z A$ & -1.74 & -4.80 \\
\hline$K P S S_{\mu}$ & $4.43^{*}$ & 0.46 \\
$K P S S_{\tau}$ & $0.32^{*}$ & 0.15 \\
\hline
\end{tabular}

Les valeurs critiques sont données à un niveau de 5\%. Pour les tests de ElliottRothenberg-Stock et ceux modifiés de Phillips-Perron, nous avons sélectionné le nombre de retards $k=17$, tandis que pour ceux de Zivot-Andrews $k=7$. Pour les tests de KPSS, nous avons sélectionné le nombre de retards $m=8$.

Tableau 2 : Détection des points de retournement Upturn correspond à la phase d'expansion, downturn à la phase de récession.

\begin{tabular}{|c|c|}
\hline Date - Upturn & Date - Downturn \\
\hline Février 1877 & Juin 1878 \\
Août-novembre 1880 & Mars 1882 \\
Octobre 1883 & Mars 1885 \\
Octobre 1888 & Septembre 1890 \\
Mai 1892 & Octobre 1893 \\
Mars 1895 & Avril 1900 \\
Mai 1902 & Juillet 1903 \\
Avril 1905 & Août 1907 \\
Février 1909 & Janvier 1910 \\
Mai 1918 & Avril 1920 \\
\hline
\end{tabular}

\section{BIBLIOGRAPHIE}

Dagum, Estela B., «A new method to reduce unwanted ripples and revisions in trend-cycle estimates from X11ARIMA », Statistics Canada, 22, 1996, pp. 77-83.

Darné, Olivier et Diebolt, Claude, «Explorations in monetary cliometrics. The Reichsbank: 1876$1920 »$, Historical Social Research, 25, 2000, pp. 23-35. 
Darné, Olivier et Diebolt, Claude, «The institutional development of the Reichsbank », Miméo, LAMETA/CNRS, Faculté des Sciences Economiques, Université Montpellier I, 2001.

Darné, Olivier et Diebolt, Claude, «A note on seasonal unit root tests », Quality \& Quantity : International Journal of Methodology, 36, 2002, pp. 305-310.

Dickey, D. A. et Fuller, W. A., « Distribution of the estimators for autoregressive time series with unit root », Journal of the American Statistical Association, 74, 1979, pp. 427-481.

Dickey, D. A. et Fuller, W. A., « Likelihood ratio statistics for autoregressive time series with unit root », Econometrica, 49, 1981, pp. 1057-1072.

Elliott, G., Rothenberg, T. J. et Stock J. H., « Efficient tests for an autoregressive unit root », Econometrica, 64, 1996, pp. 813-836.

Harvey, A. C., Forecasting, Structural Time Series Models and the Kalman Filter, Cambridge: Cambridge University Press, 1989.

Harvey, A. C., « Trend analysis », Working Paper, Faculty of Economics and Politics, University of Cambridge, 2000.

Koopman, S. J., Harvey, A. C., Doornik, J. A. et Shepard, N., Stamp 5.0. Structural Time Series Analyser, Modeller and Predictor, Chapman and Hall, 1995.

Kwiatkowski, D., Phillips, P., Schmidt, P. et Shin, Y., « Testing the null hypothesis of stationary against the alternative of a unit root: how sure are we that economic time series have a unit root? », Journal of Econometrics, 54, 1992, pp. 159-178.

Le Sage, J. P., « Analysis and development of leading indicators using a Bayesian turning-points approach », Journal of Business and Economic Statistics, 9, 1991, pp. 305-316.

$\mathrm{Ng}$, S. et Perron, P., « Lag length selection and the construction of unit root tests with good size and power », Econometrica, 69, 2001, pp. 1519-1554.

Perron, P. et Ng, S., «Useful modifications to unit root tests with dependent errors and their local asymptotic properties », Review of Economic Studies, 63, 1996, pp. 435-465.

Pfeffermann, D. et Bleuer, S. R., « Probabilistic detection of nonseasonal turning points in economic time series estimated from sample surveys », Internal report, Methodology Branch, Statistics Canada, Ottawa, 1992.

Phillips, P. C. B. et Perron, P., « Testing for unit root in time series regression », Biometrika, 75, 1988, pp. 347-353.

Zellner, A., Hong, C. et Min, C., « Forecasting turning points in international output growth rates using Bayesian exponentially weighted autoregression, time-varying parameter, and pooling techniques », Journal of Econometrics, 48, 1991, pp. 275-304.

Zivot, Eric et Andrews, D. W. K., « Further evidence on the great crash, the oil price shock and the unit root hypothesis », Journal of Business and Economic Statistics, 10, 1992, pp. 251-270. 